\title{
Quantitative magnetic resonance imaging assessments of autosomal recessive polycystic kidney disease progression and response to therapy in an animal model
}

\author{
Bernadette O Erokwu ${ }^{1}$, Christian E Anderson ${ }^{1}$, Chris A Flask ${ }^{1}$ and Katherine M Dell ${ }^{2}$
}

BACKGROUND: Autosomal recessive polycystic kidney disease (ARPKD) is associated with significant mortality and morbidity, and currently, there are no disease-specific treatments available for ARPKD patients. One major limitation in establishing new therapies for ARPKD is a lack of sensitive measures of kidney disease progression. Magnetic resonance imaging (MRI) can provide multiple quantitative assessments of the disease.

METHODS: We applied quantitative image analysis of highresolution (noncontrast) T2-weighted MRI techniques to study cystic kidney disease progression and response to therapy in the PCK rat model of ARPKD.

RESULTS: Serial imaging over a 2-month period demonstrated that renal cystic burden $(\mathrm{RCB}, \%)=$ total cyst volume (TCV)/total kidney volume $(\mathrm{TKV}) \times 100]$, TCV, and, to a lesser extent, TKV detected cystic kidney disease progression, as well as the therapeutic effect of octreotide, a clinically available medication shown previously to slow both kidney and liver disease progression in this model. All three MRI measures correlated significantly with histologic measures of renal cystic area, although the correlation of RCB and TCV was stronger than that of TKV.

CONCLUSION: These preclinical MRI results provide a basis for applying these quantitative MRI techniques in clinical studies, to stage and measure progression in human ARPKD kidney disease.

A utosomal recessive polycystic kidney disease (ARPKD) is an inherited disorder that affects $\sim 1 / 20,000$ children and is associated with significant mortality and morbidity, related to the underlying kidney and liver disease (1). Kidney disease in ARPKD patients is characterized by markedly enlarged, echogenic (bright) kidneys reflective of diffuse microscopic-collecting tubule dilatations and is usually evident at (or before) birth. End-stage kidney disease (ESKD) develops in $40-50 \%$ of affected children by the age of 15 (2). Unfortunately, there are currently no disease-specific treatments available, and clinical care is focused on standard medical management of chronic kidney disease (CKD) complications (3). Several novel therapies, however, have been shown to slow down kidney disease progression in ARPKD animal models and in adult patients with autosomal dominant PKD (ADPKD), a related "ciliopathy" (4-8).

Unfortunately, standard measures of kidney disease severity lack the sensitivity to assess ARPKD kidney disease progression over the course of a typical clinical trial. Rates of decline in glomerular filtration rate (GFR), either estimated, using serum creatinine-based measurements, or measured, using iohexol clearance studies, are variable and relatively slow, even in patients with moderate CKD (9). In addition, unlike many other forms of CKD, ARPKD patients exhibit minimal proteinuria, limiting the applicability of that marker to assess disease progression (9). This absence of clinically available methods to measure ARPKD kidney disease progression has been the most significant roadblock in developing clinical trials of novel therapies in ARPKD patients and alternative markers are needed. Volumetric-based magnetic resonance imaging (MRI) has been validated as a surrogate marker of kidney disease progression in ADPKD, a disease in which the total kidney volume (TKV) increases with time and correlates with GFR decline (10-13). In ARPKD, however, kidney size increases very early in life and then stabilizes over time, as the normal parenchyma is replaced by increasing numbers of microcysts (14). Thus, TKV measurements alone would have limited the utility in assessing kidney disease progression in ARPKD.

Quantitative MRI techniques, which can provide reproducible measurements of renal parenchymal changes, are being applied increasingly to the study of chronic kidney diseases (15-19). Several studies have utilized these techniques to assess kidney disease progression in animal models of polycystic kidney diseases and related "ciliopathies", including juvenile nephronophthisis (20-23). However, none has utilized quantitative MRI techniques to specifically assess kidney disease progression in an ARPKD model, nor have these techniques been used to measure the treatment effects of

${ }^{1}$ Department of Radiology, Case Western Reserve University, Cleveland, Ohio; ${ }^{2}$ Department of Pediatrics, Case Western Reserve University, Cleveland, Ohio. Correspondence: Katherine M. Dell (Katherine.Dell@case.edu)

Received 28 September 2017; accepted 7 February 2018; advance online publication 2 May 2018. doi:10.1038/pr.2018.24 
a clinically available therapy with the potential to target human ARPKD kidney and liver disease.

The objectives of this study in an ARPKD animal model were to (1) develop and validate an MRI biomarker of cystic kidney disease that correlates with a histologic measure of cystic kidney disease; (2) noninvasively measure kidney disease progression over time; and (3) noninvasively measure the treatment effect of a therapy known to slow down cystic kidney disease progression. To achieve these objectives, we have developed an image-analysis methodology for conventional T2-weighted (T2W) MRI techniques to study kidney disease progression and response to a therapy (i.e., octreotide) in the PCK rat, an established genetic model for ARPKD (24). This model develops hypertension and progressive kidney disease consistent with human disease (25), and several published studies have demonstrated the effectiveness of octreotide in slowing down kidney (and liver) disease progression in this model $(6,26)$.

\section{METHODS}

\section{Animal Models}

Studies were conducted in male PCK rats, an ARPKD model that arose spontaneously from a colony of Sprague-Dawley (SD) rats and carried a homozygous mutation in the rat orthologue for the human ARPKD gene, polycystic kidney and hepatic disease 1 (pkhd1) (24). PCK rats show histologic evidence of progressive kidney disease over the course of the first 6 months of life, manifested as increased renal cystic area, whereas kidney weight/body-weight ratios do not change significantly over that time period (25). PCK rats also develop hypertension, a very common feature of ARPKD kidney disease (9). Similar to ARPKD patients, the progressive kidney disease in PCK rats is not reflected in changes in standard clinical measures of progressive kidney disease (e.g., increased blood urea nitrogen and serum creatinine) until after 25 weeks of age, at which point substantial renal damage is evident (27).

Only male rats were utilized because they develop more severe kidney disease than females. Breeding pairs were obtained from a commercial vendor (Charles River Laboratories, Wilmington, MA) and the colony was maintained by homozygous breeding. Normal SD rats were also obtained from the same vendor for comparison with PCK rats. All studies were approved by the Institutional Animal Care and Use Committee (IACUC) of Case Western Reserve University and adhered to the NIH Guide for the Care and Use of Laboratory Animals.

\section{Experimental Design}

This study consisted of two parts. In the first part, our quantitative T2W-MRI methodologies for assessment of renal cystic burden (RCB), total cystic volume (TCV), and TKV were validated against histologic assessments of cystic kidney disease in a cohort of PCK rats ( $n=9$, ages 3-5 months). Age-matched SD rats were also imaged for the purposes of visual comparisons only. In the second part, a treatment trial was undertaken to assess the ability of the T2W-MRI methodology to detect kidney disease progression and the therapeutic effect of octreotide. For this treatment trial, PCK rats from within the same litters were randomly chosen for either octreotide at a dose of $20 \mathrm{mcg} / \mathrm{kg} /$ day $(n=7)$, administered by continuous subcutaneous osmotic minipump (Alzet, Cupertino, CA), or saline control $(n=6)$, also administered by minipump (6). Treatment started at the age of 1 month and ended at the age of 3 months . Animals were imaged at three time points: baseline (pretreatment, at 1 month of age), after 1 month of treatment ( 2 months of age), and after 2 months of treatment ( 3 months of age). After the final imaging session, all animals were killed for histologic analysis as outlined below.

\section{MRI Acquisition and Image Analysis}

Our goal for the quantitative MRI-methodology technique was to develop the MRI acquisition and image-analysis techniques to quantitatively assess cystic kidney disease. Each animal was anesthetized with isoflurane and placed in a prone position within a 7.0-T Bruker Biospec preclinical MRI scanner (Bruker, Billerica, $\mathrm{MA})$. The respiratory rate $(40-60$ breaths $/ \mathrm{min})$ and core body temperature $\left(35 \pm 1^{\circ} \mathrm{C}\right)$ were monitored throughout the scanning session using an integrated animal-monitoring system (SA Instruments, Stony Brook, NY). High-resolution, coronal T2W-MRI images were then acquired for each animal using a rapid acquisition with relaxation enhancement (RARE) (28) MRI-acquisition technique $(\mathrm{TR} / \mathrm{TE}=7,000 \mathrm{~ms} / 70 \mathrm{~ms}$, resolution $=400 \mu \mathrm{m} \times 400 \mu \mathrm{m}$, slice thickness $=1.5 \mathrm{~mm}$, and three signal averages). Multiple signal averages and prospective respiratory gating were used to limit respiratory motion artifacts. A total of 15-25 contiguous coronal $\mathrm{T} 2 \mathrm{~W}$ images were acquired to ensure complete coverage of each animal's kidneys. Following the MRI acquisition, all of the images were exported for off-line analysis in MATLAB (The Mathworks, Natick, MA).

A region-of-interest analysis was then performed to obtain quantitative assessments of each animal's left and right kidneys. First, the left and right kidneys were manually selected in each image to enable an assessment of the total kidney volume, TKV (in $\mathrm{mm}^{3}$ ). Care was taken to select only the renal cortex and medulla, while manually excluding the renal pelvis, renal artery, and renal vein. After determination of TKV, a manual thresholding was performed to segment the volume of cysts (hyperintense in T2W images) and the volume of a normal kidney (lower signal intensity) in each imaging slice. In order to minimize bias, the segmentation analysis was performed by a single, blinded, expert rater (CAF). These data were then compiled to determine the total $3 \mathrm{D}$ volume of renal cysts (in $\mathrm{mm}^{3}$ ) for the left and right kidneys of each animal combined. The RCB (\%), an expression of the percent (\%) of cyst volume for the left and right kidneys combined, was then calculated for each animal using the equation shown below. RCB (\%) was chosen as the means for assessing cystic kidney disease because it captures the severity of cystic kidney disease in aggregate, and would account for any variability in cystic involvement between the left and right kidneys.

$$
\begin{gathered}
\text { Renalcystic burden }(\mathrm{RCB}, \%)= \\
\frac{[\text { Total cyst volumes }(\mathrm{TCV}) \text { right }+ \text { left kidneys }]}{[\text { Total kidney volumes }(\mathrm{TKV}) \text { right }+ \text { left kidneys }]} \times 100
\end{gathered}
$$

\section{Histologic Analysis}

For both the validation and treatment portions of the study, animals were killed after the last imaging session. Kidneys were fixed in $4 \%$ paraformaldehyde and paraffin-embedded. Five-micron longitudinal sections centered at the renal hilum (right kidney) were obtained and stained with Masson's trichrome as described (25). Whole-slide sections were visualized by light microscopy, and images were captured at $\times 2$ original magnification using an attached digital camera. Images were imported into Adobe Photoshop (Adobe Systems, San Jose, CA, USA) and the cyst area in each cross section was determined by pixel counting and expressed relative to the total number of kidney pixels in the image (percent cystic area).

\section{Statistical Analysis}

TKV $\left(\mathrm{mm}^{3}\right)$, TCV $\left(\mathrm{mm}^{3}\right)$, and RCB (\%) as determined by MRI assessments outlined above were calculated for each animal at each time point. Means and standard deviations for each of the imaging measures were calculated for octreotide-treated and control animals at each time point and differences between the two groups were assessed using two-tailed Student's $t$-tests, with significance set at a $P$ 
value of $<0.05$. Pearson's correlation coefficient $(r)$ was utilized to compare the imaging and histologic variables for the initial validation cohort and the octreotide-treated and control animals after completion of the treatment protocol. Specifically, TKV, TCV, and $\mathrm{RBC}$ were each compared to the histologic assessment of percent (\%) cystic area. Significance for the correlation coefficient was set at a $P$ value of $<0.05$.

\section{RESULTS}

A total of nine cystic PCK rats underwent the initial T2W imaging studies at 3-5 months of age followed by comparison with histologic assessments. Representative images of a 3month-old PCK rat (left panel) and an age-matched SD rat (right panel) are shown in Figure 1 for comparative purposes. As expected, PCK kidneys demonstrated visibly increased T2W signal in cystic regions of the cortex and medulla consistent with macroscopic cysts (Figure 1, solid arrow). Corresponding whole-section histology is shown in the lower panels. While large, "macroscopic" cysts predominate in this model, linear streaks of increased MRI signal intensity radiating from the cortex to the medulla are also evident in
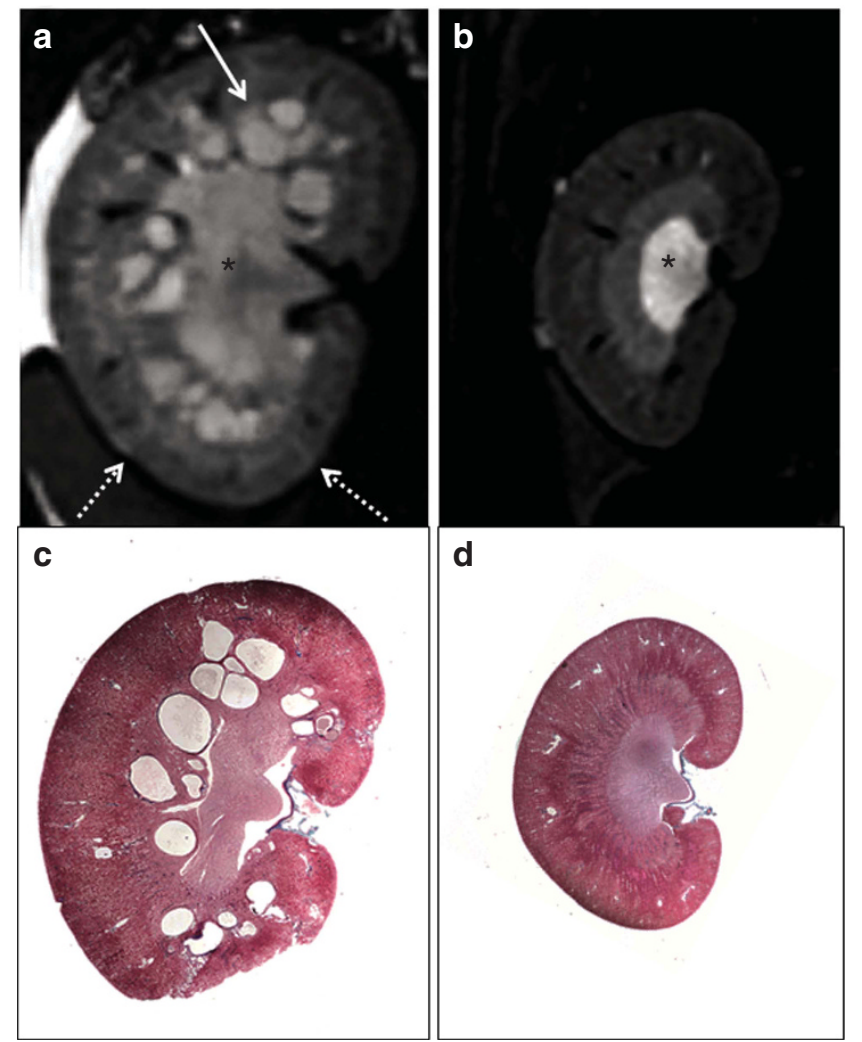

Figure 1. T2W-MRI in PCK and control rat kidneys. Representative coronal T2-weighted MRI images of the right kidneys of a 3-month-old PCK rat (a) and an age-matched Sprague-Dawley (SD) control rat (b). Discrete renal cysts are evident in the PCK kidney (solid arrow) as are smaller linear areas of hyperintensity (dashed arrows), which represent areas of microcysts seen commonly on histologic analysis of ARPKD animal models and patients. The pelvices (asterisks) are identified and segmented out during the analysis. Representative histologic cross sections of the same cystic (c) and control (d) kidneys are shown below each corresponding T2W image (Trichrome stain, $\times 2$ original magnification). MRI, magnetic resonance imaging. the full cross section of PCK rat kidney (Figure 1a, dashed arrows) and represent collections of microscopic cysts, which are often seen on histologic analysis in this model (Figure 1c and Figure 2, inset). In human ARPKD, microscopic cysts predominate but macroscopic cysts can become evident over time (14).

Figure 2 illustrates the image analysis methodology performed to quantitate the severity of cystic kidney disease as shown for a 3-month-old PCK rat. A representative T2W image with hyperintense cystic regions is shown in the left panel (Figure 2a). The region-of-interest analysis is then used to delineate the whole kidney form the surrounding anatomy and cystic regions are clearly evident on the pseudocolor map of the T2W image (Figure 2b). These maps are then manually thresholded to specifically segment the cysts from the normal renal parenchyma (Figure $2 \mathrm{c}$ ). Note that the renal pelvis, consisting primarily of urine and large renal blood vessels, was readily excluded from the analysis (Figure $\mathbf{2 b , c}$ ). This process was repeated for all imaging slices for the right and left kidneys. The inset (red box) shows a region in which both microcysts and a larger cyst are evident by T2W imaging and by corresponding histology.

RCB, TCV, and TKV for each of the nine animals in the validation studies were obtained and compared to the histologic measure of cystic kidney disease (percent cystic area). As shown in Figure 3, RCB showed a strong, significant correlation with the histologic measure (Figure 3a, $r=0.82$, $P=0.003$ ), as did TCV (Figure $3 \mathbf{b}, r=0.80, P=0.005$ ). TKV also showed a significant correlation (Figure 3c, $r=0.70$, $P=0.018$ ), although somewhat weaker than that of the other two measures.

For the treatment study, seven PCK rats underwent treatment with octreotide and six with saline control. RCB, $\mathrm{TCV}$, and TKV for the octreotide-treated and control groups at each of the imaging time points are shown in Figure $4 \mathbf{a}-\mathbf{c}$, respectively. At baseline, there were no significant differences between the treated vs. untreated animals in RCB $(10.7 \pm 4.3 \%$ vs. $\quad 11.0 \pm 3.8 \%, \quad P=0.89)$, TCV $\quad\left(138 \pm 63 \mathrm{~mm}^{3} \quad\right.$ vs. $\left.137 \pm 43 \mathrm{~mm}^{3}, \quad P=0.96\right)$ or TKV $\left(1,287 \pm 381 \mathrm{~mm}^{3}\right.$ vs. $\left.1,268 \pm 314 \mathrm{~mm}^{3}, \quad P=0.92\right)$. Both treated and untreated animals showed large increases in both TCV and TKV from baseline to 1 month. However, RCB, which reflects contributions from both of these parameters, did not increase significantly. After 1 month of treatment (2 months of age), there were no significant differences between the two treatment groups (octreotide vs. saline) in any of the imaging parameters. In contrast, after 2 months of treatment, the octreotide-treated group showed significantly lower RCB $(11.0 \pm 1.2 \%$ vs. $13.9 \pm 2.3 \%, P=0.02)$, TCV $(491 \pm 80$ vs. $\left.709 \pm 130 \mathrm{~mm}^{3}, \quad P=0.01\right)$ and TKV $(4,463 \pm 411$ vs. $\left.5,085 \pm 288 \mathrm{~mm}^{3}, \quad P=0.01\right)$ when compared with salinetreated controls. Figure 5 shows the RCB of individual rats for each time point (Figure $\mathbf{5 a}$, octreotide treated, Figure $\mathbf{5 b}$, saline control). Consistent with human disease, some animals show relatively stable RCB (e.g., gray line in each figure), whereas others, especially in the saline control group, show a 

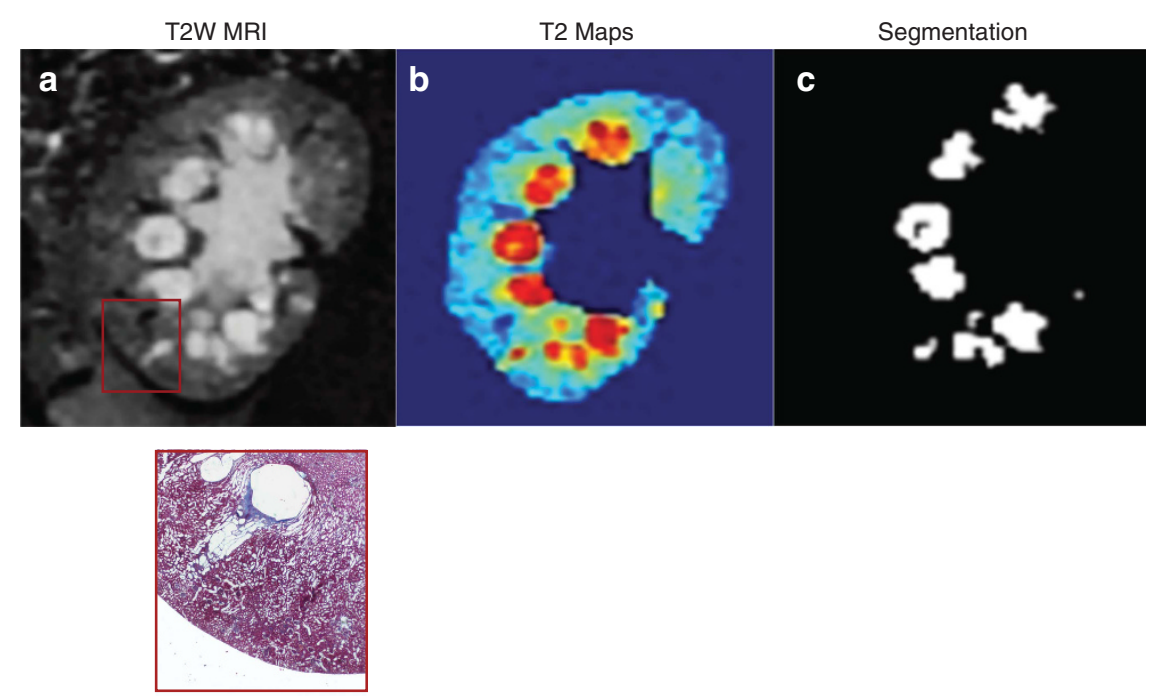

Figure 2. Thresholding analysis of a PCK rat kidney. (a) Representative coronal T2W-MRI images of the right kidney from a 3-month-old PCK rat. (b) Pseudocolor intensity map (arbitrary units) showing medullary and cortical cysts/cystic regions in red with normal renal parenchyma in blue. (c) Cystonly map obtained by manual thresholding of the T2W images. Note the agreement between the cyst map (c) and the hyperintense cystic regions in the T2W image (a). The inset (red box) shows a region in which both microcysts and a larger cyst are evident by T2W imaging as confirmed by corresponding histology (Trichrome stain, $\times 2$ original magnification).
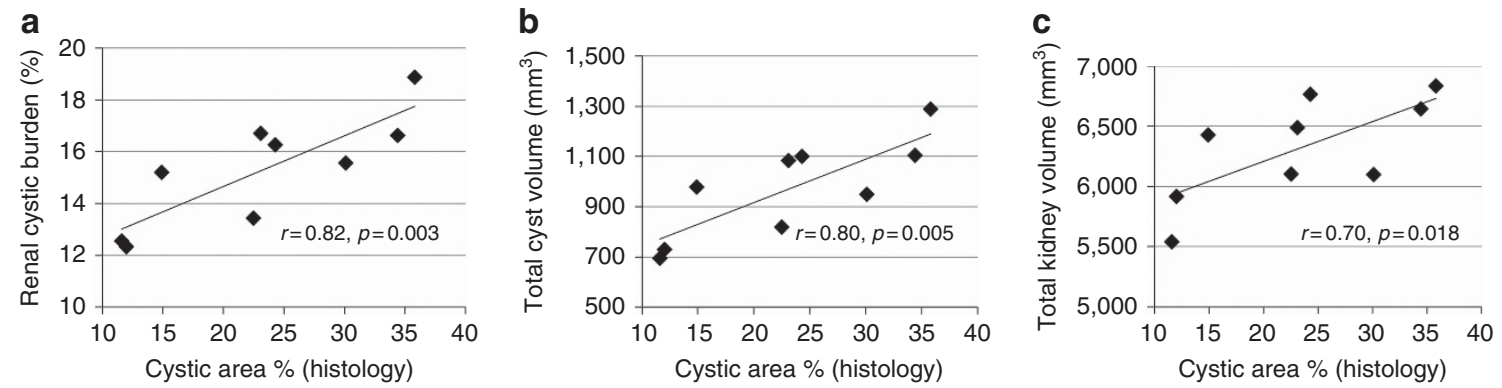

Figure 3. Validation of MRI assessments of cystic kidney disease in PCK rats. Scatterplots demonstrating the relationship between histologic assessments (percent cystic area, \%) and the MRI assessments of: (a) RCB (\%); (b) TCV $\left(\mathrm{mm}^{3}\right)$ of the left and right kidneys; and (c) TKV (mm $\left.{ }^{3}\right)$ of the left and right kidneys for the 3-5-month-old PCK rats studied $(n=9)$. All three imaging parameters showed significant correlations with histologic measures of percent cystic area, with the strongest correlations seen with TCV and RCB. ( $r=$ Pearson correlation coefficient, $P=$ significance level). MRI, magnetic resonance imaging; RCB, renal cystic burden; TCV, total cyst volume; TKV, total kidney volume.
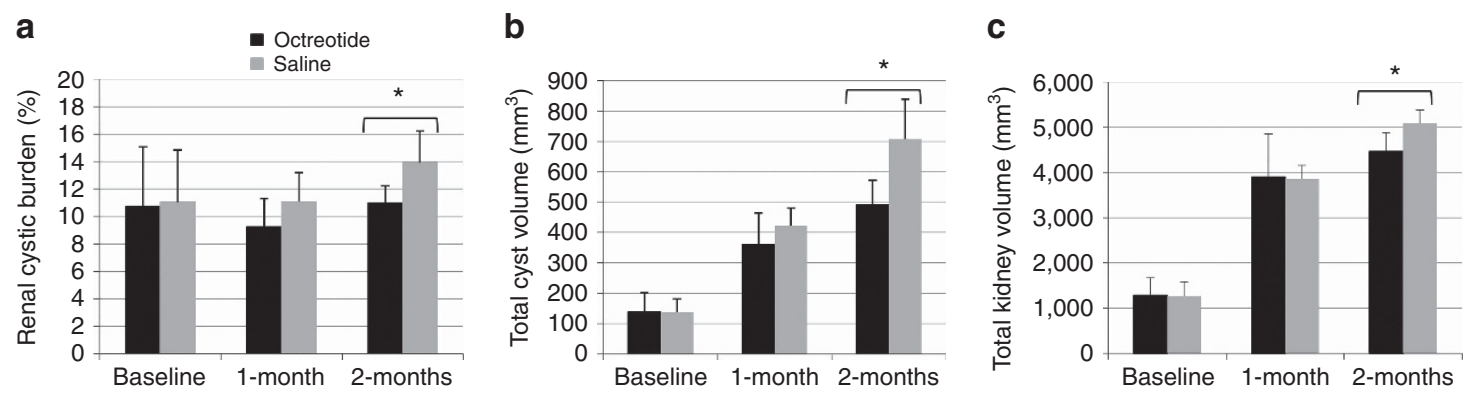

Figure 4. Longitudinal kidney MRI assessment of cystic kidney disease in octreotide-treated and control rats. MRI measures of (a) RCB (\%), (b) TCV $\left(\mathrm{mm}^{3}\right)$ and $(\mathbf{c})$ TKV $\left(\mathrm{mm}^{3}\right)$ for octreotide-treated and saline-treated PCK rats scanned at baseline (1 month of age) and 1 and 2 months after starting octreotide or saline control ( 2 and 3 months of age, respectively). No significant differences were noted in any of the imaging parameters at baseline or after 1 month of treatment. However, all three parameters (RCB, TCV, and TKVs) detected significant differences in octreotide-treated compared to saline controls after 2 months of therapy $(P=0.025, P=0.007, P=0.009$, respectively). MRI, magnetic resonance imaging; RCB, renal cystic burden; $\mathrm{TCV}$, total cyst volume; TKV, total kidney volume. 


\section{T2 MRI of ARPKD progression}
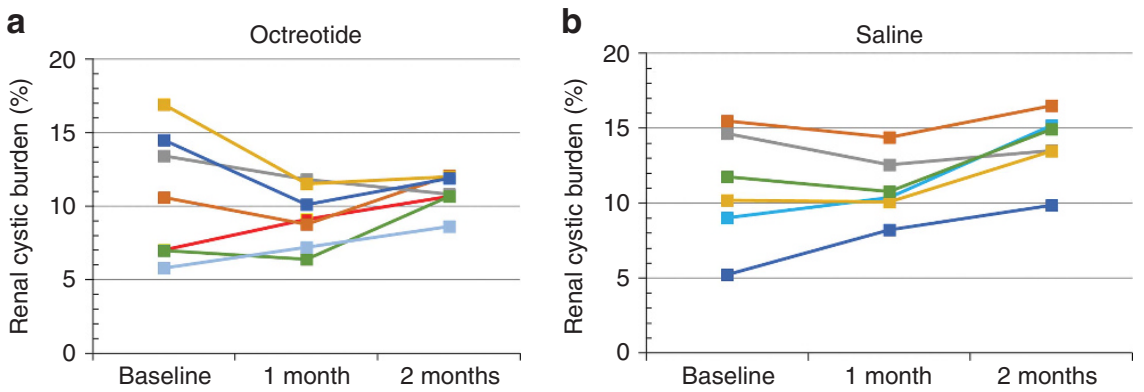

Figure 5. Changes in RCB over time. RCB for (a) octreotide-treated and (b) saline-treated PCK rats at each study time point (baseline, 1 month and 2 months) demonstrates the ability of MRI to detect not only changes in the groups as a whole, but also the variable rates of cystic kidney disease progression in individual animals. MRI, magnetic resonance imaging; RCB, renal cystic burden.
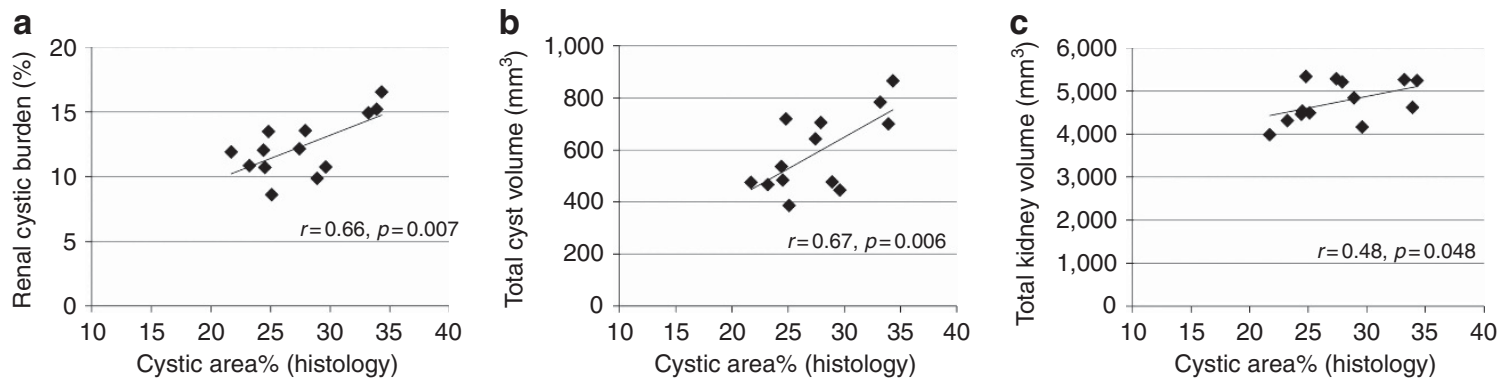

Figure 6. Comparisons of MRI and histologic assessments of cystic renal disease. Scatterplot of histological assessments (\% cystic area) compared to (a) RCB; (b) TCV; and (c) TKV. RCB and TCV correlated significantly strongly with \% cystic area. TKV showed a weaker, but significant correlation as well. ( $r=$ Pearson correlation coefficient, $P=$ significance level). MRI, magnetic resonance imaging; RCB, renal cystic burden; TCV, total cyst volume; TKV, total kidney volume.

more rapid increase (e.g., blue and green lines in Figure 5b). Interestingly, several of the octreotide-treated animals showed a decrease in RCB between baseline and 1 month (e.g., yellow and blue lines, Figure 5a). However, as a group this difference did not reach significance $(P=0.4)$.

Histologic assessment of octreotide compared saline-treated animals after 2 months of therapy showed a significant difference between the two (mean \pm SD for cystic area percent: octreotide $=25.1 \pm 2.6$; control $=30.5 \pm 3.9, P=0.02$ ), consistent with past reports $(6,26)$. As was done in the initial validation studies (Figures 1,2,3), we also examined the relationship of $\mathrm{RCB}, \mathrm{TCV}$, and $\mathrm{TKV}$ to the histologic assessments of cystic kidney disease for the octreotide and saline-treated animals (Figure 6). All three measures showed significant correlation with histology. However, the correlations for RCB $(r=0.66, P=0.007)$ and TCV $(r=0.67, P=0.006)$ were stronger than that of $\mathrm{TKV}$, which just reached significance $(r=0.48, P=0.048)$.

\section{DISCUSSION}

In the current study, we utilized T2W-MRI techniques to measure changes in RCB, TCV, and TKV over time and in response to therapy in an ARPKD animal model. Using a manual thresholding approach, we demonstrated that $\mathrm{T} 2 \mathrm{~W}$ MRI imaging can be used to sensitively measure cystic kidney disease progression and response to octreotide in the PCK rat over a 2-month period. Specifically, RCB, TCV, and TKV all detected therapeutic effects during the time period studied. In addition, RCB and TCV showed significant correlations with histologic assessments of cystic kidney disease. TKV also showed a significant correlation, although not as strong as the other two imaging biomarkers. Several previous studies have applied quantitative MRI techniques to the study of kidney disease progression (and response to therapy); however, these studies have focused on genetic models of other forms of PKD, specifically juvenile nephronophthisis $(21,22)$, a disorder that is clinically distinct from ARPKD. Ours is the first study to systematically apply quantitative image analysis of T2W-MRI techniques to assess kidney disease progression and response to therapy in a genetic model of ARPKD.

Although a very limited number of MRI studies of human ARPKD kidney disease have been reported, these focused primarily on volumetric and/or qualitative assessments and have not quantified changes in the severity of cystic kidney disease over time (29,30). The T2W-MRI acquisition and analysis described in this paper has a number of advantages to enable future clinical MRI research studies in ARPKD patients. The hyperintense cystic regions in the T2W images provide an obvious choice to delineate the renal cystic changes from the normal kidney parenchyma (Figures 1 and 2). In addition, T2W-MRI acquisitions can be performed using a variety of rapid MRI imaging techniques (e.g., 


\section{Articles | Erokwu et al.}

HASTE, Half-Fourier Acquisition Single Shot Turbo Spin Echo) that could be obtained in a single breathhold $(<10 \mathrm{~s})$ on virtually any MRI scanner. The use of rapid MRI acquisition techniques limits the effects of respiratory motion artifacts on the final kidney assessments. As such, these rapid MRI acquisitions would provide very short overall MRI scan times for ARPKD patients ( $<5$ min of scan time), potentially limiting the need for sedation and anesthesia in younger ARPKD patients. In addition, MRI does not utilize ionizing radiation and, in the case of several MRI assessments (including the $\mathrm{T} 2 \mathrm{~W}$ imaging utilized in this study), no intravenous gadolinium contrast agent is required. The latter is important when considering MRI studies in chronic kidney disease (CKD) since gadolinium chelates have been implicated in the development of nephrogenic systemic fibrosis (NSF) in patients with advanced CKD (31). Quantitative MRI methodologies, including T2w imaging, also provide significant advantages over other imaging modalities, notably ultrasonography, in terms of reproducibility and the ability to measure changes in cystic and normal parenchyma over time.

$\mathrm{RCB}$, as assessed by T2W-MRI, has particular advantages in the study of ARPKD, a disease that disproportionately affects younger children. During childhood, kidneys normally grow in concert with linear growth. Early in ARPKD, kidneys typically enlarge rapidly, but then kidney growth slows as the remaining normal parenchyma is replaced with cysts. As noted previously, unlike ADPKD, kidney size in ARPKD stabilizes over time, despite ongoing disease progression (14). Utilizing TCV or TKV alone as MRI biomarkers of disease progression may under or overestimate the actual severity of cystic involvement, depending on the relative growth of normal or cystic parenchyma in a given child. In contrast, RCB captures the proportion of cystic involvement in relationship to the size of the kidneys and would be anticipated to more accurately reflect disease progression through childhood. The translational possibilities of using RCB (in addition to TCV or TKV alone) are well illustrated in our preclinical study. These include the ability to noninvasively measure the effect of a therapy in treated vs. control subjects (e.g., Figure 4) and noninvasively assess kidney disease progression in an individual subject or groups of subjects in aggregate (e.g., Figure 5). In addition, these assessments would allow identification of "high risk" subjects (e.g., those with high RCB and/or rapidly increasing RCB) who may benefit the most from therapeutic interventions.

It is also important to note that our study noninvasively measured the treatment effect of octreotide, which, unlike some of the other novel therapies (such as vasopressin receptor antagonists), has been used for other indications in pediatric patients. This medication also has the potential to impact both kidney and liver disease, as shown in previous treatment studies in this model $(6,26)$ and in studies of human ADPKD (32). In separate work, we are identifying and optimizing imaging biomarkers for ARPKD liver disease progression (33). Thus, our preclinical findings in cystic kidney disease progression in the current study, coupled with ongoing ARPKD liver disease imaging biomarker development, would provide the basis for potentially rapidly translatable studies. Importantly, clinical trials could determine whether the efficacy of octreotide in slowing cystic kidney disease (and liver disease) progression demonstrated in animal studies translates into a long-term impact on kidney function decline or liver disease progression in ARPKD patients.

Our study did have several limitations. First, the PCK rat develops a slowly progressive form of ARPKD kidney disease with a predominance of macrocysts rather than the typical microcysts evident at an early age in human ARPKD. However, the imaging was able to detect both micro and macrocysts (Figure 2). And, notably, macrocysts in ARPKD patients do occur and can become more prominent during childhood (14). Thus, although the PCK rat does not fully phenotypically mirror kidney disease in the subset of subjects with very rapidly progressive disease resulting in end-stage kidney disease in infancy, it may reflect the more slowly progressing patients who reach end-stage kidney disease in later childhood or even adulthood. Second, the detection of renal cysts via T2W-MRI imaging does require manual segmentation, which could be subject to variations based on expertise and number of personnel performing the analysis. In order to minimize this potential variability, all of the segmentation analysis was performed by only one expert rater for this initial study. Quantitative MRI techniques that generate quantitative $\mathrm{T} 1$ and $\mathrm{T} 2$ relaxation time maps may also be used to facilitate automatic, more objective segmentation of the kidneys and renal cysts $(34,35)$. However, these quantitative acquisitions typically require extended acquisition times, limiting their utility for young pediatric ARPKD patients. Even with rapid ( $<5 \mathrm{~min}) \mathrm{T} 2 \mathrm{w}$ imaging acquisition times, such as those described in this paper, accurate scans may be difficult to obtain in children, primarily related to motion artifact. As a result, young children typically require sedation and/or anesthesia in order to undergo MRIs. To address this issue, in separate studies, our group is currently exploring the application of novel magnetic resonance fingerprinting techniques pioneered in our institution, which can simultaneously provide quantitative $\mathrm{T} 1$ and $\mathrm{T} 2$ relaxation time maps in $\sim 10$ s/imaging slice and are inherently resistant to motion artifact $(36,37)$. The relaxometric MRI results described in this manuscript suggest that these magnetic resonance fingerprinting techniques could eventually provide a practical, rigorously quantitative MRI approach for pediatric ARPKD patients. Third, we noted considerable variability in disease severity in the animals studied, despite the fact that the PCK breeding colony is maintained by homozygous breeding and the animals studied were littermates from only a limited number of litters. However, significant treatment effect was still found despite these variations. Furthermore, as noted above, this phenotypic variation is also seen in human ARPKD kidney disease. Thus, the MRI findings in the animal model may more reliably 
reflect true disease (compared to measures such as serum creatinine), and allow for identification of patients who are at higher risk for progression. Finally, T2W-MRI may not fully capture other aspects of progressive CKD, namely tubulointerstitial microstructure changes that reflect tubulointerstitial damage (fibrosis). The application of other quantitative MRI methodologies, such as diffusion-weighted MRI, to the study of renal fibrosis is an active area of investigation by our group and others $(19,38)$.

In summary, the results of this study suggest that quantitative image analysis of T2W-MRI images can sensitively detect kidney disease progression and response to therapy in ARPKD. These preclinical results set the stage for application of these techniques to studies of human ARPKD.

\section{ACKNOWLEDGMENTS}

We thank Dr Tatyana Masyuk for guidance regarding the octreotide treatment protocol as well as Mr Jose Mariappurum and Dr Ying Gao for technical assistance.

\section{STATEMENT OF FINANCIAL SUPPORT}

This work was supported by NIH/NIDDK R01-DK085099 (Co-PIs:Dell/Flask).

Disclosure: The authors declare no conflict of interest.

\section{REFERENCES}

1. Dell KM. The spectrum of polycystic kidney disease in children. Adv Chronic Kidney Dis 2011;18:339-47.

2. Roy S, Dillon MJ, Trompeter RS, et al. Autosomal recessive polycystic kidney disease: long-term outcome of neonatal survivors. Pediatr Nephrol 1997;11:302-6.

3. Guay-Woodford LM, Bissler JJ, Braun MC, et al. Consensus expert recommendations for the diagnosis and management of autosomal recessive polycystic kidney disease: report of an international conference. J Pediatr 2014;165:611-7.

4. Gattone VHn, Wang X, Harris PC, et al. Inhibition of renal cystic disease development and progression by a vasopressin V2 receptor antagonist. Nat Med 2003;9:1323-6.

5. Sabbatini M, Russo L, Cappellaio F, et al. Effects of combined administration of rapamycin, tolvaptan, and AEZ-131 on the progression of polycystic disease in PCK rats. Am J Physiol Renal Physiol 2014;306: F1243-50.

6. Masyuk TV, Radtke BN, Stroope AJ, et al. Pasireotide is more effective than octreotide in reducing hepatorenal cystogenesis in rodents with polycystic kidney and liver diseases. Hepatology 2013;58: 409-21.

7. Torres VE, Chapman AB, Devuyst O, et al. Tolvaptan in patients with autosomal dominant polycystic kidney disease. N Engl J Med 2012;367: 2407-18.

8. Cramer MT, Guay-Woodford LM. Cystic kidney disease: a primer. Adv Chronic Kidney Dis 2015;22:297-305.

9. Dell KM, Matheson M, Hartung EA, et al. Kidney disease progression in autosomal recessive polycystic kidney disease. J Pediatr 2016;171: 196-201.e1.

10. O'Neill WC, Robbin ML, Bae KT, et al. Sonographic assessment of the severity and progression of autosomal dominant polycystic kidney disease: the Consortium of Renal Imaging Studies in Polycystic Kidney Disease (CRISP). Am J Kidney Dis 2005;46:1058-64.

11. Chapman AB, Guay-Woodford LM, Grantham JJ, et al. Renal structure in early autosomal-dominant polycystic kidney disease (ADPKD): the Consortium for Radiologic Imaging Studies of Polycystic Kidney Disease (CRISP) cohort. Kidney Int 2003;64:1035-45.
12. Bae KT, Grantham JJ. Imaging for the prognosis of autosomal dominant polycystic kidney disease. Nat Rev Nephrol 2010;6:96-106.

13. Cadnapaphornchai MA, Masoumi A, Strain JD, et al. Magnetic resonance imaging of kidney and cyst volume in children with ADPKD. Clin J Am Soc Nephrol 2011;6:369-76.

14. Avni FE, Guissard G, Hall M, et al. Hereditary polycystic kidney diseases in children: changing sonographic patterns through childhood. Pediatr Radiol 2002;32:169-74.

15. Xu X, Fang W, Ling H, et al. Diffusion-weighted MR imaging of kidneys in patients with chronic kidney disease: initial study. Eur Radiol 2010;20: 978-83.

16. Zhao J, Wang ZJ, Liu M, et al. Assessment of renal fibrosis in chronic kidney disease using diffusion-weighted MRI. Clin Radiol 2014;69: $1117-22$.

17. Zheng Z, Shi H, Zhang J, et al. Renal water molecular diffusion characteristics in healthy native kidneys: assessment with diffusion tensor MR imaging. PLoS ONE 2014;9:e113469.

18. Zhang JL, Morrell G, Rusinek H, et al. New magnetic resonance imaging methods in nephrology. Kidney Int 2014;85:768-78.

19. Lu L, Sedor JR, Gulani V, et al. Use of diffusion tensor MRI to identify early changes in diabetic nephropathy. Am J Nephrol 2011;34:476-82.

20. Ta MH, Schwensen KG, Foster S, et al. Effects of TORC1 inhibition during the early and established phases of polycystic kidney disease. PLoS ONE 2016;11:e0164193.

21. Franke M, Baessler B, Vechtel J, et al. Magnetic resonance T2 mapping and diffusion-weighted imaging for early detection of cystogenesis and response to therapy in a mouse model of polycystic kidney disease. Kidney Int 2017;92:1544-54.

22. Ta MH, Rao P, Korgaonkar M, et al. Pyrrolidine dithiocarbamate reduces the progression of total kidney volume and cyst enlargement in experimental polycystic kidney disease. Physiol Rep 2014;2:e12196.

23. Aihara M, Fujiki H, Mizuguchi $H$, et al. Tolvaptan delays the onset of end-stage renal disease in a polycystic kidney disease model by suppressing increases in kidney volume and renal injury. J Pharmacol Exp Ther 2014;349:258-67.

24. Ward CJ, Hogan MC, Rossetti S, et al. The gene mutated in autosomal recessive polycystic kidney disease encodes a large, receptor-like protein. Nat Genet 2002;30:259-69.

25. Goto M, Hoxha N, Osman R, et al. The renin-angiotensin system and hypertension in autosomal recessive polycystic kidney disease. Pediatr Nephrol 2010;25:2449-57.

26. Masyuk TV, Masyuk AI, Torres VE, et al. Octreotide inhibits hepatic cystogenesis in a rodent model of polycystic liver disease by reducing cholangiocyte adenosine 3',5'-cyclic monophosphate. Gastroenterology 2007;132:1104-16.

27. Mason SB, Liang Y, Sinders RM, et al. Disease stage characterization of hepatorenal fibrocystic pathology in the PCK rat model of ARPKD. Anat Rec 2010;293:1279-88.

28. Hennig J, Nauerth A, Friedburg H. RARE imaging: a fast imaging method for clinical MR. Magn Reson Med 1986;3:823-33.

29. Gunay-Aygun M, Font-Montgomery E, Lukose L, et al. Correlation of kidney function, volume and imaging findings, and PKHD1 mutations in 73 patients with autosomal recessive polycystic kidney disease. Clin J Am Soc Nephrol 2010;5:972-84.

30. Liebau MC, Serra AL. Looking at the (w)hole: magnet resonance imaging in polycystic kidney disease. Pediatr Nephrol 2013;28:1771-83.

31. Fraum TJ, Ludwig DR, Bashir MR, et al. Gadolinium-based contrast agents: a comprehensive risk assessment. J Magn Reson Imaging 2017;46: 338-53.

32. Ruggenenti P, Remuzzi A, Ondei P, et al. Safety and efficacy of longacting somatostatin treatment in autosomal-dominant polycystic kidney disease. Kidney Int 2005;68:206-16.

33. Gao Y, Erokwu BO, DeSantis DA, et al. Initial evaluation of hepatic T1 relaxation time as an imaging marker of liver disease associated with autosomal recessive polycystic kidney disease (ARPKD). NMR Biomed 2016;29:84-9. 


\section{Articles | Erokwu et al.}

34. Nieto JA, Yamin MA, Goldberg ID, et al. An empirical biomarker-based calculator for cystic index in a model of autosomal recessive polycystic kidney disease-the Nieto-Narayan formula. PLoS ONE 2016;11: e0163063.

35. Kim Y, Ge Y, Tao C, et al. Automated segmentation of kidneys from MR images in patients with autosomal dominant polycystic kidney disease. Clin J Am Soc Nephrol 2016;11:576-84.
36. Gao Y, Chen Y, Ma D, et al. Preclinical MR fingerprinting (MRF) at 7 T: effective quantitative imaging for rodent disease models. NMR Biomed 2015;28:384-94.

37. Ma D, Gulani V, Seiberlich N, et al. Magnetic resonance fingerprinting. Nature 2013;495:187-92.

38. Morrell GR, Zhang JL, Lee VS. Magnetic resonance imaging of the fibrotic kidney. J Am Soc Nephrol 2017;28:2564-70. 